\title{
Discrimination and Quantification of Fe and Ni Abundances in Genesis Solar Wind Implanted Collectors using X-ray Standing Wave Fluorescence Yield Depth Profiling with Internal Referencing
}

\author{
Y. Choi ${ }^{1}$, P. Eng ${ }^{2,3}$, J. Stubbs ${ }^{2}$, S. R. Sutton ${ }^{2,4^{*}}$, M. Schmeling ${ }^{5}$, \\ I. V. Veryovkin ${ }^{6}$ and D. Burnett ${ }^{7}$ \\ ${ }^{1}$ X-ray Science Division, Building 431E, 9700 S. Cass Avenue, Advanced Photon \\ Source, Argonne, IL 60439 USA \\ ${ }^{2}$ Center for Advanced Radiation Sources, University of Chicago, Building 434A, 9700 S. \\ Cass Avenue, Argonne, IL 60439 USA \\ ${ }^{3}$ James Franck Institute, University of Chicago, Chicago, Illinois 60439, USA \\ ${ }^{4}$ Department of Geophysical Sciences, 5734 S. Ellis Ave, University of Chicago, \\ Chicago, IL 60637 USA \\ ${ }^{5}$ Department of Chemistry and Biochemistry, 1032 W. Sheridan Rd., Loyola University, \\ Chicago, IL 60660 USA \\ ${ }^{6}$ Department of Chemistry, University of Illinois at Chicago, Chicago, IL 60607 USA \\ ${ }^{7}$ Division of Geological and Planetary Sciences, MS 100-23, California Institute of \\ Technology, Pasadena, CA 91125 USA
}

* Corresponding author. Email: sutton@cars.uchicago.edu

Submitted to Chemical Geology

May 2016

Revision submitted August 2016 


\begin{abstract}
X-ray standing wave fluorescence yield depth profiling was used to determine the solar wind implanted Fe and Ni fluences in a silicon-on-sapphire (SoS) Genesis collector (60326). An internal reference standardization method was developed based on fluorescence from $\mathrm{Si}$ and $\mathrm{Al}$ in the collector materials. Measured Fe fluence agreed well with that measured previously by us on a sapphire collector (50722) as well as SIMS results by Jurewicz et al. Measured Ni fluence was higher than expected by a factor of two; neither instrumental errors nor solar wind fractionation effects are considered significant perturbations to this value. Impurity Ni within the epitaxial Si layer, if present, could explain the high $\mathrm{Ni}$ fluences and therefore needs further investigation. As they stand, these results are consistent with minor temporally-variable $\mathrm{Fe}$ and $\mathrm{Ni}$ fractionation on the timescale of a year.
\end{abstract}

Keywords: Solar wind fluence; Genesis mission; x-ray standing wave analysis; depth profile modeling; implant quantification 


\section{Introduction}

The Genesis spacecraft, launched by NASA in 2001, spent 850 days orbiting the Earth-Sun L1 Lagrange point collecting solar wind by implantation into a variety of pure collector materials, including sapphire, gold, silicon and diamond-like carbon. The mission science goal was to improve the accuracy of chemical and isotopic abundances for the solar photosphere by making solar wind samples available for analysis by sophisticated laboratory instruments. Such improved accuracy is crucial in further developing models of solar evolution and processes.

Although the spacecraft's transport to L1, the solar wind collection activities and its 2004 transit to Earth were highly successful, the reentry vehicle containing the sample return capsule failed to deploy its parachute resulting in a high velocity impact in the Utah desert, fragmentation of the collector wafers and severe contamination of the surfaces of the ultra-pure materials with terrestrial matter. The subsequent challenge has been to nonetheless realize the scientific promise of the Genesis mission by developing analytical techniques designed to allow discrimination between terrestrial surface contamination and implanted solar wind. These efforts have involved development and testing of surface cleaning methodologies followed by application of surface sensitive analytical techniques, such as secondary ion mass spectrometry (SIMS) and resonance ionization mass spectrometry (RIMS). A formidable challenge for the surface cleaning applications is to remove the contamination from the surfaces without affecting the implanted solar wind ions residing at depths on the order of $100 \mathrm{~nm}$, 
For quantification of implanted solar wind, SIMS and RIMS are the two surface sensitive techniques that have been applied successfully (e.g., Burnett 2011 and 2013; Heber et al. 2014; Veryovkin et al. 2011). Although these techniques are destructive in that the implanted species plus the collector substrate are sputtered away with the energetic primary ions, the analysis footprint (sputtered crater) is relatively small ( hundreds of microns) compared to the available area of the collector fragments ( a few $\mathrm{mm}$ ) thus permitting many independent analyses on the same collectors at different locations. Much of the recent SIMS and RIMS effort has focused on improving analytical reproducibility and accuracy by minimizing sputter depth profiling artifacts originating from ion mixing phenomena (e.g., Sigmund and Gras-Marti 1980).

The purpose of this paper is to describe the application of the $\mathrm{x}$-ray standing wave phenomenon to the non-destructive quantification of solar wind implanted fluences. The x-ray standing wave (XSW) technique at grazing incident angles has been a valuable tool in characterizing the trace element distribution in thin film samples (e.g., Becker et al. 1983, Bloch et al. 1985, Wang et al. 1992, Dev et al. 2000, Templeton et al. 2001, Lee et al. 2006, Gupta et al. 2007, Tiwari et al. 2008, Ghose et al. 2001, Kitts et al. 2009). XSW makes use of the interference between incoming and outgoing grazing x-rays resulting in a nodal pattern of electric field intensity localized perpendicular and near to the surface (Becker et al. 1983; Bloch et al. 1985). By measuring the angular dependence of the fluorescence of the implanted elements and taking advantage of the localized electric field enhancement due to this interference, depth profiles and subsequently quantitative elemental abundance of implanted ions can be determined. An advantage of this method compared to SIMS and RIMS is that depth information extracted from x-ray 
reflectivity curves allows the positions of buried structural interfaces to be determined and, therefore, surface contamination to be distinguished from implanted species. A novel aspect of this work is the use of the fluorescence data for a major substrate element as an internal reference for quantification of the other detectible elements.

\section{Methodology}

\subsection{Samples}

The flight samples studied in this work were (1) sapphire $\left(\mathrm{Al}_{2} \mathrm{O}_{3}\right)$ labeled $\mathrm{S} 50722$ and (2) silicon-on-sapphire (SoS) fragment labeled SoS60326, kindly provided by the Genesis Solar Wind Sample Curator (J. Allton) at Johnson Space Center, Houston, TX. The results for the sapphire sample were described by Kitts et al. (2009). This paper will focus on the SoS sample. SoS samples are particularly advantageous for these measurements because the air-silicon and silicon-sapphire interfaces can be readily identified in x-ray reflectivity profiles and therefore provide valuable depth markers for data interpretation (see below).

60326 is a silicon-coated, single sapphire crystal $9.3 \mathrm{~mm}$ in length by $22.7 \mathrm{~mm}$ wide by $0.7 \mathrm{~mm}$ thick, a total solar wind exposed area of $138 \mathrm{~mm}^{2}$. The silicon coating, $\sim 2000 \AA$ A thick, was epitaxially grown on a pre-formed, polished, crystal (Jurewicz et al. 2003). Prior to analysis, 60326 was flushed with ultra-pure water to remove large surface particulates and cleaned in a UV/ozone instrument to remove organic contamination without disturbing the bulk substrate (Calaway et al. 2007); both procedures were performed at the curatorial facility. 
In addition to the flight sample, the analysis methodology was applied to two laboratory implantation standards: (1) sapphire $\left(\mathrm{Al}_{2} \mathrm{O}_{3}\right)$ flight spare implanted with $2 \times 10^{13}{ }^{56} \mathrm{Fe}^{+} / \mathrm{cm}^{2}$ (courtesy of B. Rout, University of North Texas, Denton, TX), (2) SoS flight spare implanted with $3 \times 10^{13}$ ions $/ \mathrm{cm}^{2}$ of both ${ }^{55} \mathrm{Mn}^{+}$and ${ }^{60} \mathrm{Ni}^{+}$at 55 and $60 \mathrm{keV}$, respectively (Leonard Kroko, Inc, Tustin $\mathrm{Ca}$ ). The quoted implant fluences for these standards are considered nominal values and significant uncertainties (up to 50\%) may exist (Burnett et al. 2015).

\subsection{Reflectivity, X-ray Standing Wave, and Fluorescence Yield Measurements}

The flight samples and laboratory-implanted spare were analyzed using the Newport General Purpose Diffractometer (Trainor et al. 2006) at the GSECARS sector 13 at the Advanced Photon Source (APS; Argonne National Laboratory). The x-ray beam was derived from an APS undulator with the gap set to supply $11.5 \mathrm{keV}$ photons at the undulator fundamental. A cryogenic Si (111) double-crystal monochromator was used to narrow the energy bandwidth of the beam. A combination of focusing mirrors in a Kirkpatrick-Baez geometry and slits resulted in a 20 x $500 \mu \mathrm{m}(\mathrm{H} \mathrm{x} \mathrm{V)} \mathrm{x-ray} \mathrm{beam}$ containing $\sim 10^{12}$ photons $/ \mathrm{sec}$. At the grazing incident angles used, the actual footprint of the beam on the sample exceeds $8 \mathrm{~mm}$, representing $\sim 8 \times 0.5 \mathrm{~mm}$ area over which the XSW measurements average.

The samples and spares were mounted on the diffractometer with the surface normal horizontal and enclosed in a helium-flow sample chamber containing a thin (5 $\mu \mathrm{m})$ Kapton window. A downstream ionization detector was used to measure the intensity of the reflected x-ray beam. A Vortex ME-4, silicon-drift, energy-dispersive 
array detector (SII NanoTechnology Inc., now Hitachi High-Technologies Science America, Inc.), mounted with its detector elements parallel to the sample surface, was used to collect XRF spectra as a function of incident angles of the x-ray beam on the sample surface, adjusted using the diffractometer. Silver foil shielding and a custom detector collimator - in direct contact with the Kapton window - were used to limit the detector's view to a restricted area of the sample surface.

The critical angle of reflection $\left(\theta_{\mathrm{C}}\right)$, the maximum angle at which $\mathrm{x}$-rays are reflected from an interface, provides a valuable depth marker. $\theta_{\mathrm{C}}$ for both air-silicon and silicon-sapphire interfaces were determined from x-ray reflectivity curves obtained using specular reflection $(\theta-2 \theta)$ trajectory scans with an angular sampling interval of 4 millidegrees $(\theta)$. Full XRF spectra, collected at each of these angles, form the basis for the quantification of implant fluences.

The individual XRF spectra were processed via a peak-fitting routine. The XRF spectra from the four detector elements were averaged and corrected for dead time. The total areas of fluorescence peaks were fit using Gaussian line shapes after subtracting the background. The resulting net fluorescence peak areas were then plotted against incident angle to produce a fluorescence yield profile for each detected element between 1 and 8.8 $\mathrm{keV}$. The element responsible for each XRF peak was identified using tabulated energies of electron transitions (Krause and Oliver 1979). For Al and Si, the K $\alpha$ plus $\mathrm{K} \beta$ peaks were used while the Ka peaks were used for all others. 


\subsection{X-ray Standing Wave Theory}

The grazing incidence XSW technique is a combined approach between grazing incidence reflectivity and fluorescence measurements. Specular x-ray reflectivity, with the same incident and reflecting angles, is sensitive to the electron density profile in the sample depth direction. The specular reflectivity can be analyzed using Parratt's (1954) recursive formalism with a depth profile of refractive index $n$. For a fixed incident x-ray wavelength $\lambda$, the refractive index at a certain depth position can be written as,

$$
n=1-\delta+i \beta=1-\frac{\lambda^{2} r_{e} n_{a t}}{2 \pi}\left(f^{\prime}-i f^{\prime \prime}\right)
$$

where $r_{e}$ is the classical electron radius, $n_{a t}$ the atomic density of the element, and $f$," the real and imaginary parts of atomic scattering factors that depend on $\lambda . \delta$ and $\beta$ are real and imaginary correction terms to account for dispersion and absorption, respectively. For example, the refractive indices for the $\mathrm{Al}_{2} \mathrm{O}_{3}$ substrate can be written as,

$$
n=1-\frac{\lambda^{2} r_{e}}{2 \pi}\left[n_{a t, A l}\left(f_{A l}^{\prime}-i f_{A l}^{\prime \prime}\right)+n_{a t, O}\left(f_{O}^{\prime}-i f_{O}^{\prime \prime}\right)\right]
$$

Grazing incidence XSW makes use of the interference between incident and reflected grazing $\mathrm{x}$ rays. Near the critical angle of total external reflection $\left[\theta_{\mathrm{C}}=(2 \delta)^{0.5}\right]$, the interference results in enhanced electric field intensity variation localized near the surface. Using parameters obtained from the reflectivity analysis, the corresponding electric field intensity variation can be calculated as a function of x-ray incident angle $\theta$ and depth $z$ (Parratt 1954, de Boer 1991). X-ray fluorescence intensity modulation depends on the local electric field intensity $|E(\theta, z)|^{2}=I_{E F}(\theta, z)$ and depth profile of 
fluorescing element concentration $N(z)$. The fluorescence yield $Y$, normalized to the incident x-ray beam flux, can be written as (Bedzyk et al. 1988, 1989),

$$
Y(\theta)=\text { Const } \int_{z_{\min }}^{z_{\max }} I_{E F}(\theta, z) N(z) e^{-z / l_{a}} G(\theta) d z
$$

where $l_{a}(=\lambda / 4 \pi \beta)$ is the attenuation length of emitted fluorescence. Const is an x-ray energy-, element-, and setup-dependent constant, and $G(\theta)$ is a footprint correction factor to account for the x-ray beam footprint change with angle on the sample surface. As seen in the equations above, the fluorescence and reflectivity analysis is based on one dimensional modeling (as a function of $z$ only), thus providing laterally averaged information.

\subsection{Substrate Reference Fluorescence}

The x-ray fluorescence intensity measured from the major substrate element is used to calibrate the signals from the other trace (implanted) elements. This approach relies on the fact that the substrates are single crystals with well-defined structures and stoichiometries. The number of $\mathrm{Al}$ atoms per unit volume in the sapphire substrate, for

example, can be readily calculated to be $4.690 \times 10^{22} \mathrm{Al} / \mathrm{cm}^{3}$. This value is $n_{a t, A l}$ in Eq. 1a. The same set of structural parameters from the reflectivity analysis can be applied to the XSW fluorescence analysis. The variable $z_{\max }$ in Eq. 2 is the entire substrate thickness, however, for computational simplicity, an appropriate maximum depth $z_{\max }$ is chosen, taking advantage of the fact that the incident $\mathrm{x}$-rays fail to penetrate completely through substrate at the small grazing incident angles of interest here. 


\subsection{Elemental Dependence of Measured Fluorescence Intensities}

In order to use the substrate fluorescence for absolute quantification, there are additional factors related to the fluorescence emission and detection processes that need to be added to Eq. 2. Figure 1 summarizes these processes which depend on the incident $\mathrm{X}$-ray energy and element. The detected fluorescence intensities [for example, I(Al-K $\alpha)$ and $\mathrm{I}(\mathrm{Fe}-\mathrm{K} \alpha)$ from the $\mathrm{Fe}: \mathrm{Al}_{2} \mathrm{O}_{3}$ sample] are proportional to the incident $\mathrm{x}$-ray intensity

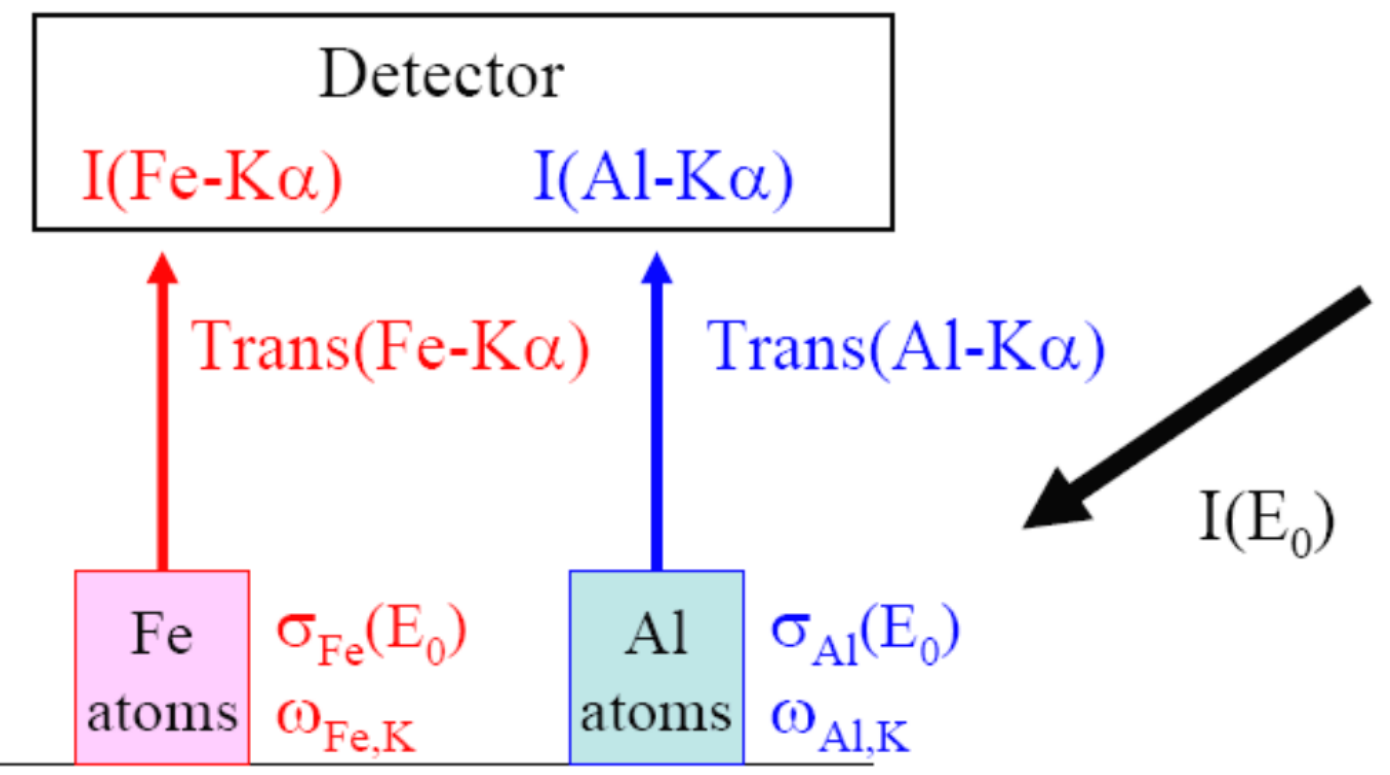

Figure 1: Schematics summarizing the element and energy dependent factors included in the $\mathrm{Fe}: \mathrm{Al}_{2} \mathrm{O}_{3}$ data analysis. $\mathrm{I}\left(\mathrm{E}_{0}\right), \mathrm{I}(\mathrm{Al}-\mathrm{K} \alpha)$, and $\mathrm{I}(\mathrm{Fe}-\mathrm{K} \alpha)$ represent the incident $\mathrm{x}$-ray and measured $\mathrm{Al}$ and Fe fluorescence intensities, respectively. $\sigma$ and $\omega$ represent photoelectric cross section at the incident $\mathrm{x}$-ray energy $\mathrm{E}_{0}$ and fluorescence yield for K fluorescence, respectively. For each fluorescence, Trans represents the transmission ratio from the sample surface to the detector, including the detector efficiency at the fluorescence energy. $G$ and $C_{G e o}$ represent geometrical factors that are common for the simultaneously measured $\mathrm{Al}$ and Fe fluorescence. $G$ depends on the sample size, the incident x-ray beam size, and incident angle. $C_{G e o}$ depends on the detector setup. 
$\mathrm{I}\left(\mathrm{E}_{0}\right)$, element-dependent photoelectric cross-section $\sigma_{\text {photo-e }}\left(E_{0}\right)$, fluorescence yield $\omega_{K}$, and transmission ratios between the sample surface and the detector. The absorption of an x-ray by the sample depends on the atom and the incident x-ray energy $E_{0}$. This dependence is expressed as the photoelectric cross-section $\sigma_{\text {photo-e }}\left(E_{0}\right)=2 r_{e} \lambda f^{\prime \prime}$, neglecting small coherent and incoherent scattering cross-section contributions for moderate x-ray energies. Once an atom absorbs an incident x-ray creating an electron hole in the process, the excited atom can relax either by the non-radiative Auger electron process or by the fluorescence emission process. Each element has characteristic fluorescence yields $\left(\omega_{K}\right)$ for K-, L-, and M-shells. For example, for K-shell, $\omega_{K}$ quantifies the relative probability for a $\mathrm{K}$ fluorescence versus Auger electron emission. Overall, $\sigma_{\text {photoee }}\left(E_{0}\right)$ (in Barns/atom) and $\omega_{K}$ (unitless) increase as atomic number $Z$ increases, and thus these two factors favor fluorescence emission from high $Z$ elements. These two factors are well tabulated for non-resonant energies (Chantler 1995, 2000; Elam et al. 2002; Krause 1979; McMaster et al. 1969), allowing comparative analysis between fluorescence intensities from trace and substrate elements. The product of these two intrinsic factors is expressed as $C_{I n t}$ in the calculations (Table 1). Since each fluorescence yield value describes all the fluorescence lines from a given shell, all the fluorescence lines need to be considered (e.g., $\omega_{K}$ includes $\mathrm{K}_{\alpha 1}, \mathrm{~K}_{\alpha 2}$ and $\mathrm{K}_{\beta}$ ). However, in practice, only the most intense lines are measured (e.g., $\mathrm{K}_{\alpha 1}$ and $\mathrm{K}_{\alpha 2}$ ) in which case a correction factor for the transition probability is necessary. Each fluorescence emission has a characteristic energy dependent on the electron energy levels involved, and thus the fluorescence emissions from different elements undergo different degrees of attenuation between the emission point in the sample and the 
detector. As mentioned above, the attenuation within the sample is included in Eq. 2. The additional attenuation to the fluorescence detector can be readily determined if the distance and materials between the sample surface and the fluorescence detector are known. The net transmission factor for each $\mathrm{K} \alpha$ fluorescence radiation is expressed as $C_{E x t}$. In addition, the fluorescence detector efficiency, which depends on fluorescence energy, is included in $C_{E x t}$. As an example, $C_{\text {Int }}$ and $C_{E x t}$ values in the measurements are summarized in Table 1. Taking into account the intrinsic and extrinsic factors mentioned above, Const in Eq. 2 now consists of Const $=C_{\text {Int }} \times C_{E x t} \times C_{G e o}$, where $C_{\text {Int }}$ and $C_{E x t}$ represent intrinsic and extrinsic elemental dependence, respectively. The constant $C_{G e o}$ is an energy-independent geometrical factor accounting for the solid angle of fluorescence detection.

\subsection{Depth Profile Modeling}

In order to extract the total number of implanted atoms, e.g., determine the solar wind fluence, the measured fluorescence yield profile is fit to equation (2) to obtain $\mathrm{N}(\mathrm{z})$ and then $N(z)$ is integrated over depth. The fitting of $N(z)$ is accomplished by assuming a model depth profile and optimizing its magnitude, depth and width. Various depth profiles were attempted and it was found that the total fluence results (proportional to the area under the profile) were quite insensitive to the precise shape of the profile used indicating that the method is better at determining total fluences than precise implant 


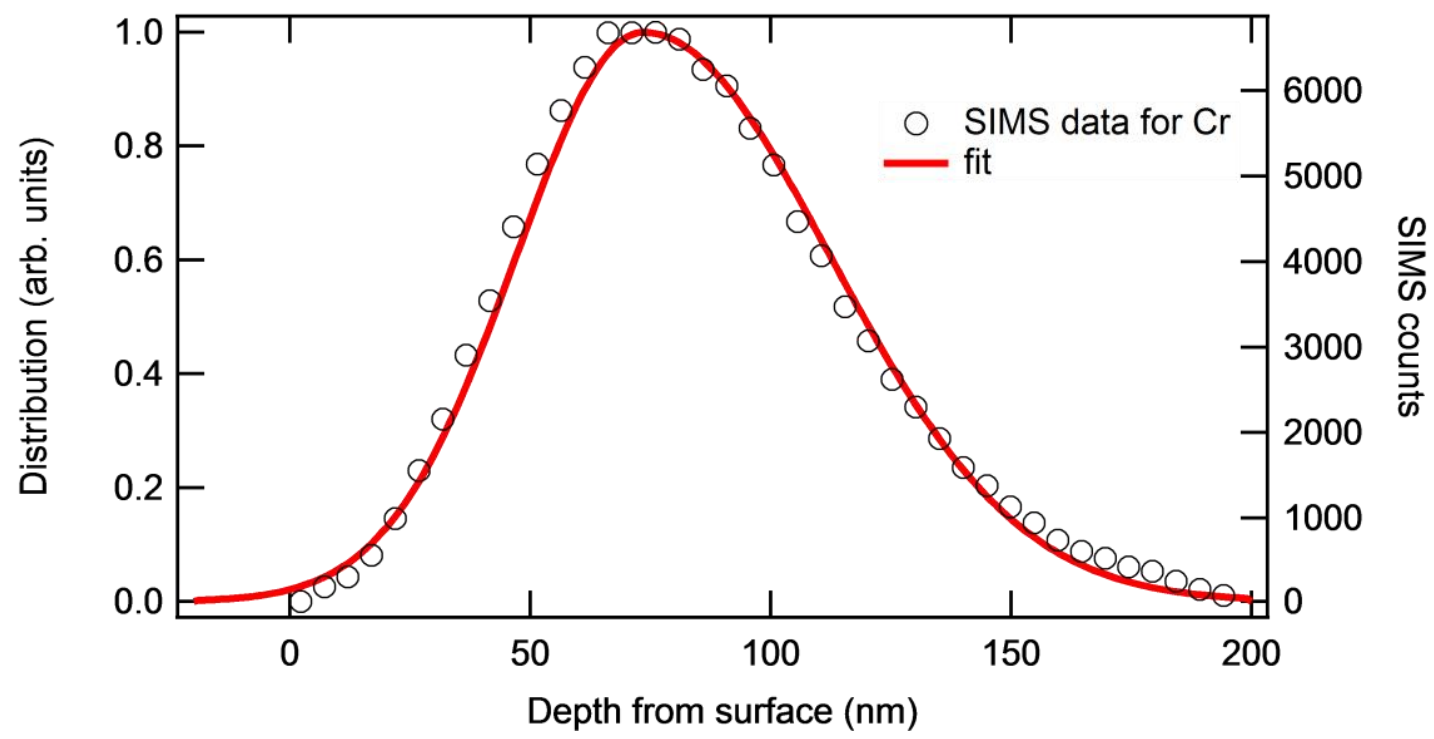

Figure 2: Depth profile used to fit depth distribution of implanted atoms. Points are data for solar wind implanted $\mathrm{Cr}$ measured by SIMS. The line is a functional fit to the SIMS data based on an asymmetric Gaussian (see text).

depth distributions.

The principal depth profile used in this work to define the distribution of an implant was that derived from SIMS analysis of solar wind $\mathrm{Cr}$ from Genesis flight sample SoS 01863 . This asymmetric profile, consisting of 40 discrete measurement points distributed over $200 \mathrm{~nm}(50 \AA / \mathrm{pt})$, was approximated by an analytical expression produced by two separate Gaussian functions of different widths using the left half of the narrow Gaussian and the right half of the wider one. The approximating function is given by:

$$
I(z)=s e^{-\left(z-z_{0}\right) /\left(a w^{2}\right)}
$$

where $s$ is a scaling factor, $z$ is depth, $z_{0}=720 \mathrm{~nm}, w$ is $550 \mathrm{~nm}$ and $a$ is an asymmetry factor which is 0.68 for $z<z_{0}$ and 1 for $z \geq z_{0}$. Figure 2 shows the comparison between the SIMS data points and this approximating function. In addition to the implant profile, a 
symmetric Gaussian function was also fit to the fluorescence yield profile to account for "at surface" contamination.

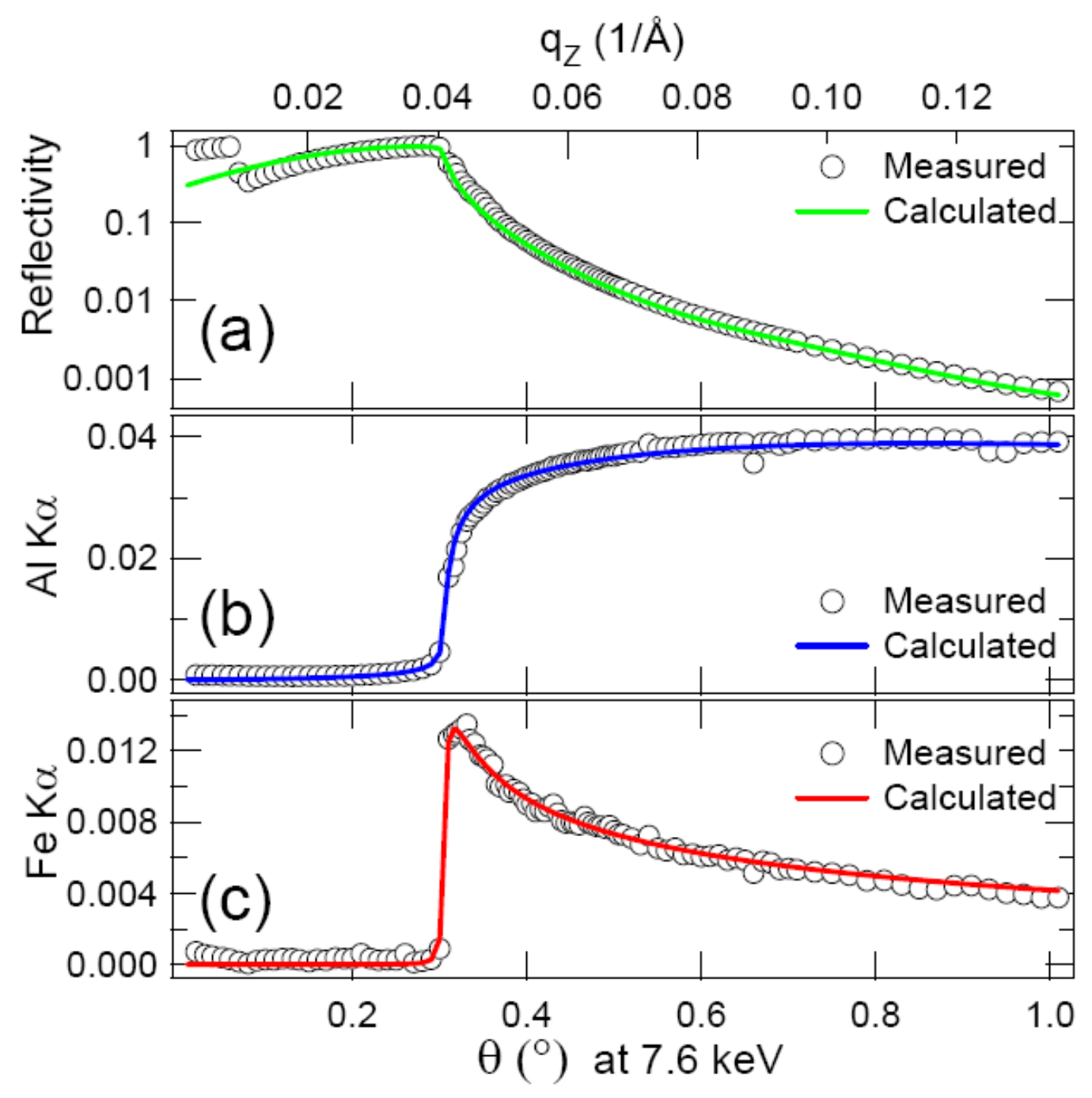

Figure 3: Reflectivity, $\mathrm{Al}$, and $\mathrm{Fe}$ fluorescence curves from the $\mathrm{Fe}: \mathrm{Al}_{2} \mathrm{O}_{3}$ sample. Reflected and fluorescence intensities were normalized to the incident $\mathrm{X}$-ray beam flux. (a) Reflectivity. (b) $\mathrm{Al} \mathrm{K \alpha}$. (c) $\mathrm{Fe} \mathrm{K \alpha}$. 


\section{Results}

\section{1 $\mathrm{Fe}: \mathrm{Al}_{2} \mathrm{O}_{3}$ Implant Standard}

Reflectivity and fluorescence curves from the $\mathrm{Fe}: \mathrm{Al}_{2} \mathrm{O}_{3}$ sample are shown in Fig. 3. The substrate density from the reflectivity fit in Figure 3(a) is the same as that of the nominal $\mathrm{Al}_{2} \mathrm{O}_{3}$ structure, and thus the $\mathrm{Al}$ atom distribution $N_{A l}(z)$ can be determined as shown in Figure 4(a). Based on the parameters from the reflectivity, $I_{E F}(\theta, z)$ is calculated. Here the electric field intensity variation is rather simple since there is only one reflecting surface. In the reflectivity and electric field intensity calculations, the presence of the implanted Fe atoms is assumed negligible. This assumption is justified because, even if all of the implanted Fe atoms are confined within a volume of $1 \mathrm{~cm}^{2} \times 1 \AA$, the Fe concentration $\left(2 \times 10^{13} \mathrm{Fe} / \mathrm{cm}^{2} \AA \hat{)}\right)$ is still small compared with the substrate $\mathrm{Al}$

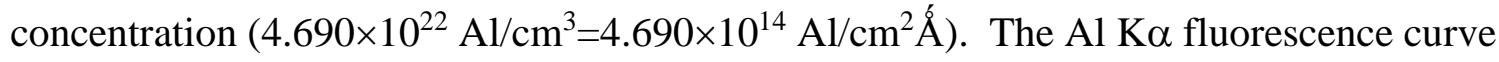
is fitted by varying $C_{G e o}$ as a scaling factor while using $N_{A l}(z)$ in Figure 4(a), $C_{I n t, A l K \alpha}$ and $C_{E x t, A l K \alpha}$. The calculated $\mathrm{Al} \mathrm{K \alpha}$ fluorescence curve is in good agreement with the measured as shown in Figure 3(b). Once the substrate Al fluorescence is fitted, quantitative analysis of the Fe fluorescence can be done. The $\mathrm{Al}$ and Fe fluorescence curves were measured simultaneously so that the $C_{G e o}$ is the same for both. Next, $C_{I n t, F e}$ $K \alpha$ and $C_{E x t, F e K \alpha}$ can be determined since the energies of the incident X-ray and fluorescence emissions are known. Consequently, the only unknown in Eq. 2 is $N_{F e}(z)$ 


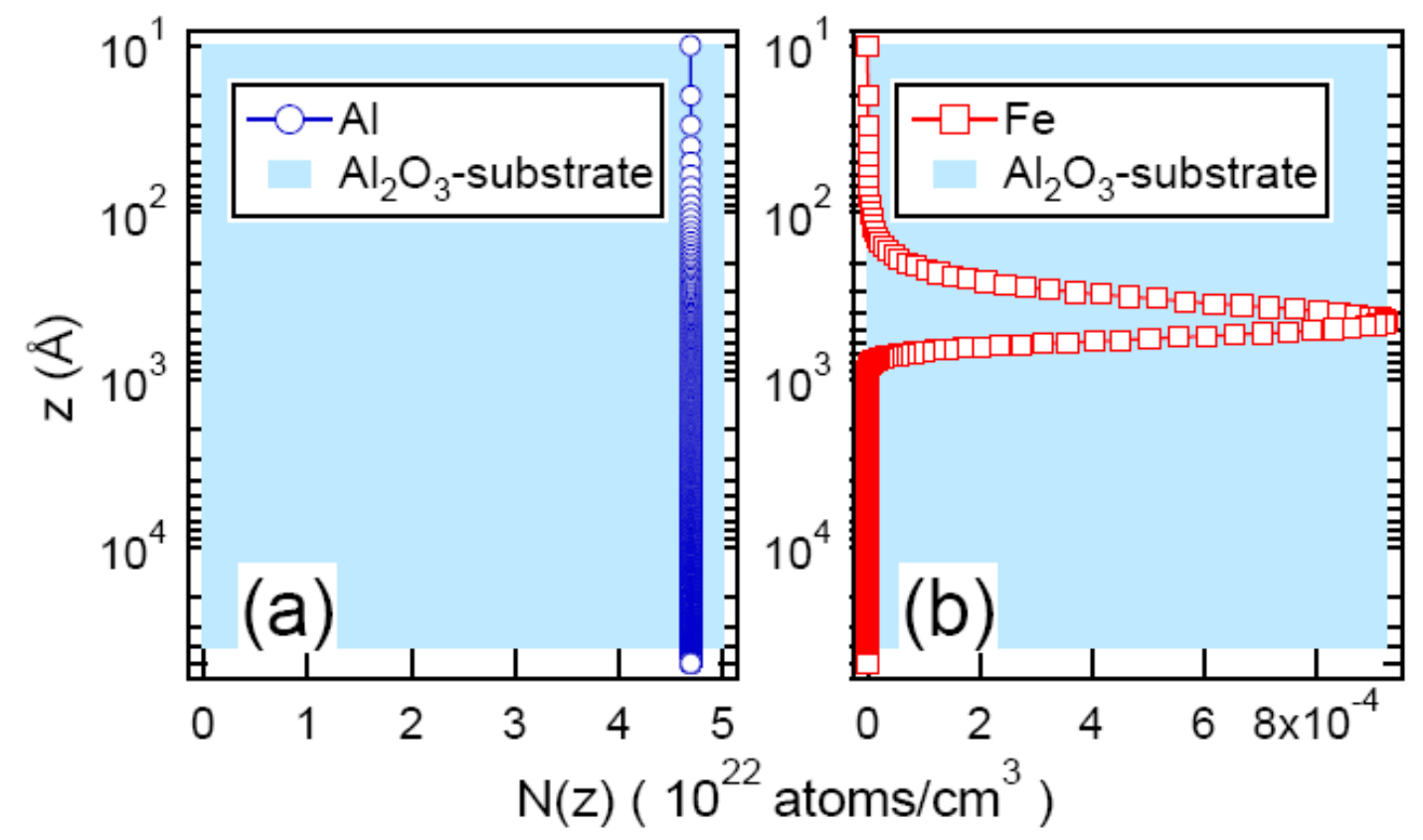

Figure 4: Elemental depth profiles for the $\mathrm{Fe}: \mathrm{Al}_{2} \mathrm{O}_{3}$ sample. (a) $\mathrm{Al}$ depth profile obtained from the x-ray reflectivity analysis in Fig. 3(a) and used to calculate the XSW result in Fig. 3(b). (b) Fe depth profile, modeled with a Gaussian peak, to fit the XSW results in Fig. 3(c).

that has the same units as $N_{A l}(z) . N_{F e}(z)$ is modeled with a Gaussian peak, and Figure 4(b) shows fitted $N_{F e}(z)$ that is centered at $449 \AA$. The integrated area under the fitted $N_{F e}(z)$ provides $2.5 \pm 0.45 \times 10^{13} \mathrm{Fe} / \mathrm{cm}^{2}$ which is consistent with the nominal value $2 \times 10^{13}$ $\mathrm{Fe} / \mathrm{cm}^{2}$

\subsection{Mn,Ni:Si: $\mathrm{Al}_{2} \mathrm{O}_{3}$ Implant Standard}

The measured reflectivity curve from the Mn and Ni implanted silicon-on-sapphire (SoS) sample is shown in Figure 5 (black curve). The separate critical angles for the airsilicon interface and the silicon-sapphire interface are clearly observed and define the location of the implant-bearing silicon layer in angle-space unmistakably, i.e., 0.21-0.27 deg. The reflectivity fit (Figure 5, red curve) yielded the structural profile shown in the 
Figure 5 inset consisting of: (1) $2.8 \mathrm{~nm}$ RMS roughness on the external Si surface, (2) 9.8 $\mathrm{nm}$ thick Si layer of density $2.19 \mathrm{~g} / \mathrm{cm}^{3}$ (optimized density and thickness), (3) $267 \mathrm{~nm}$ thick Si layer of density $2.33 \mathrm{~g} / \mathrm{cm}^{3}$ and a bulk $\mathrm{Al}_{2} \mathrm{O}_{3}$ substrate of density $3.97 \mathrm{~g} / \mathrm{cm}^{3}$ (nominal densities assumed). The substrate density agrees well with the nominal sapphire density

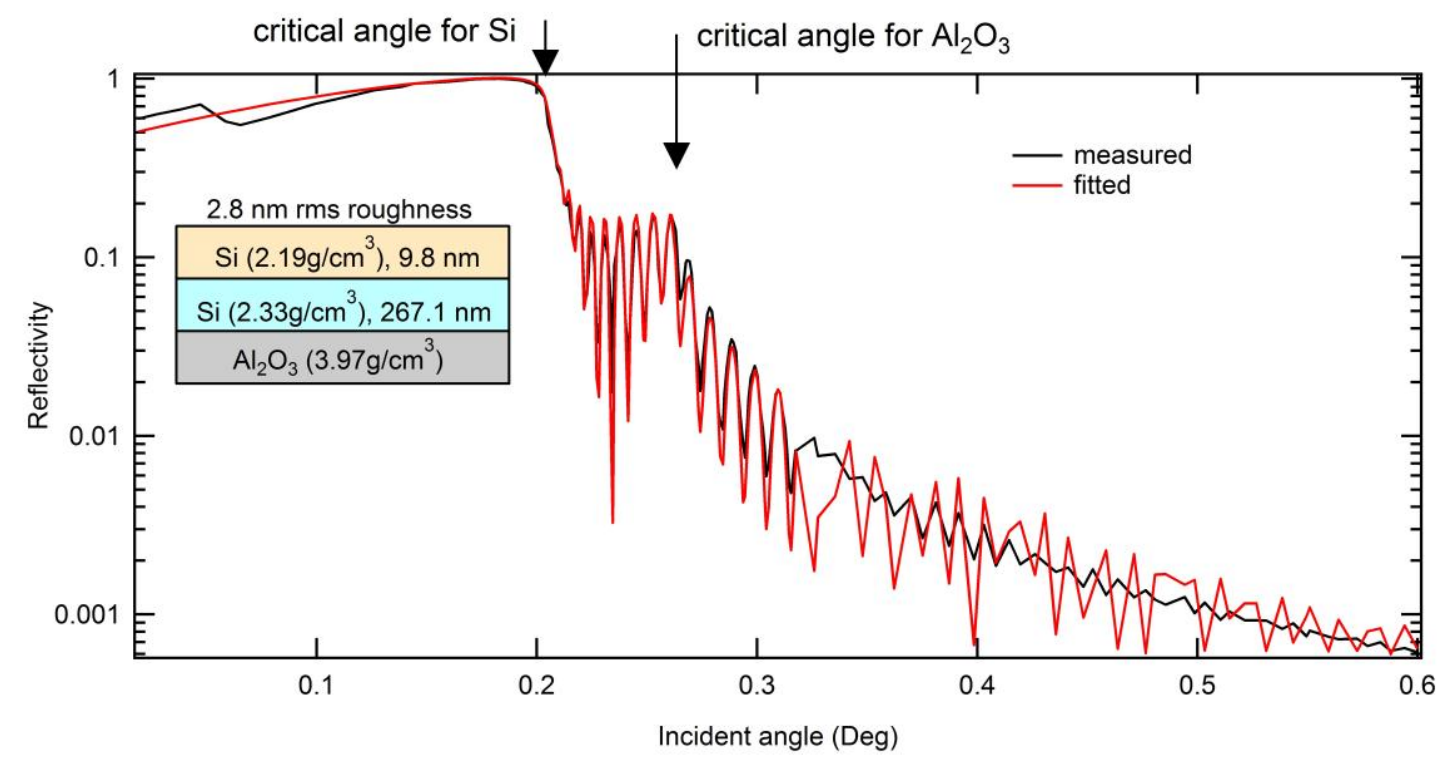

Figure 5: Reflectivity curves from the $\mathrm{Mn}$ and $\mathrm{Ni}$ implanted silicon-on-sapphire $($ SOS $)$; measured $=$ black, fitted $=$ red. The separate critical angles for the air-silicon interface and the silicon-sapphire interface are clearly observed and define the location of the implant-bearing silicon layer in angle-space unmistakably, i.e., $0.21-0.27 \mathrm{deg}$. The reflectivity fit yielded the structural profile shown in the inset consisting of: (1) $2.8 \mathrm{~nm}$ RMS roughness on the external Si surface, (2) $9.8 \mathrm{~nm}$ thick Si layer of density $2.19 \mathrm{~g} / \mathrm{cm}^{3}$, (3) $267 \mathrm{~nm}$ thick Si layer of density $2.33 \mathrm{~g} / \mathrm{cm}^{3}$ and a bulk $\mathrm{Al}_{2} \mathrm{O}_{3}$ substrate of density $3.97 \mathrm{~g} / \mathrm{cm}^{3}$.

as does the density of the thicker Si layer. The $\sim 10$-nm thick, low-density, top layer corresponds to an external oxidized layer of silica, also observed $(3.5 \mathrm{~nm})$ by spectroscopic ellipsometry (McNamara and Stansbery 2005). The total Si thickness (267 $\mathrm{nm}$ ) agrees well with the nominal thickness of $300 \mathrm{~nm}$ (Jurewicz et al. 2003). 

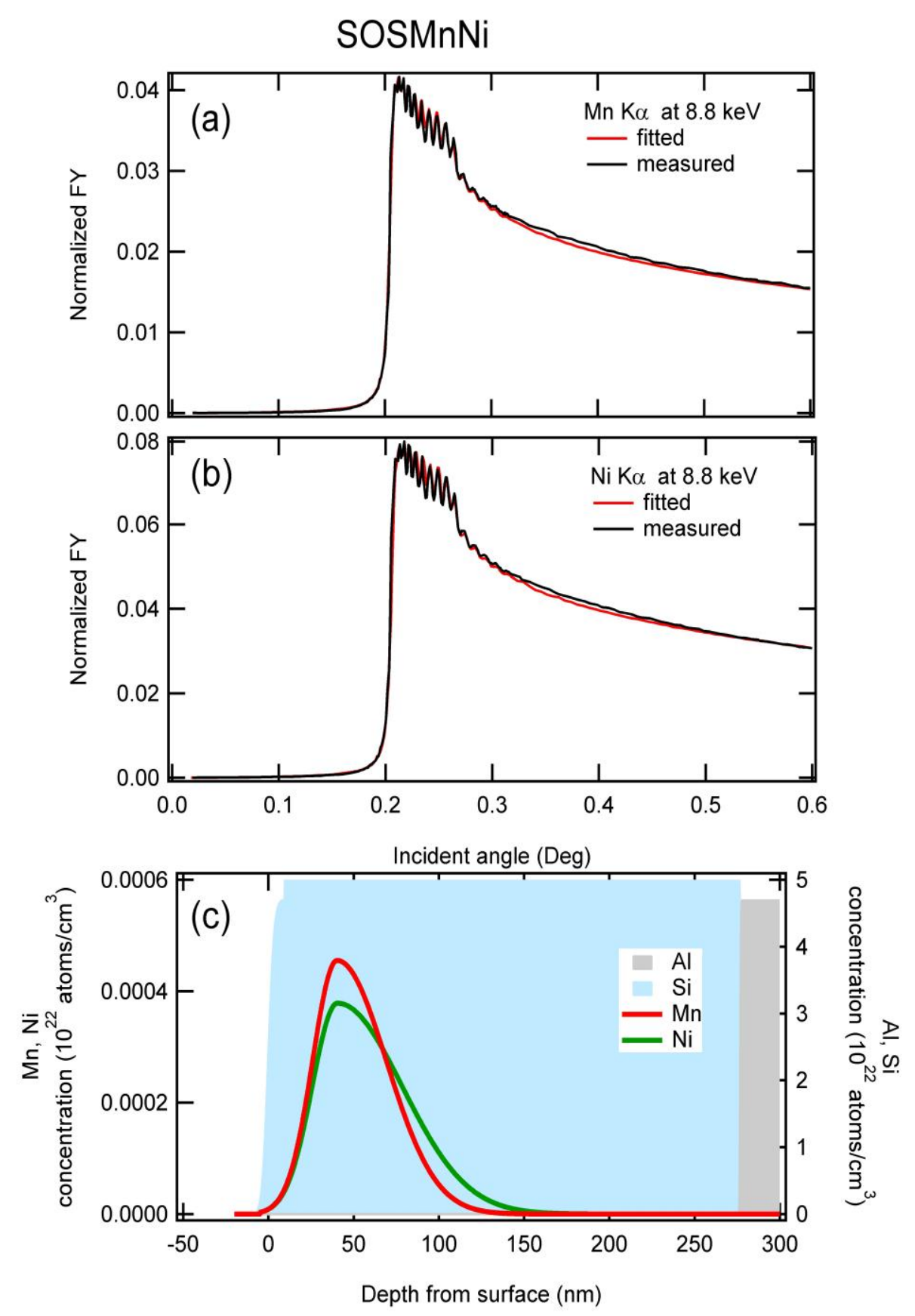

Figure 6: Measured (black curves) and fit (red curves) fluorescence yield profiles for $\mathrm{Mn} \mathrm{K} \alpha$ and $\mathrm{Ni} \mathrm{K} \alpha, \mathrm{A}$ and $\mathrm{B}$, respectively, in the implanted SoS. The sharp rise in both profiles at the air-silicon critical angle $(0.21 \mathrm{deg})$ indicates the high cleanliness of the exterior surface of the silicon layer. Fig. 5c shows the associated depth profile for the four elements obtained with the same approach as for the Fe-implant. 
Figs. 6A and 6B show the measured (black curves) and fit (red curves) fluorescence yield profiles for $\mathrm{Mn} \mathrm{K} \alpha$ and $\mathrm{Ni} \mathrm{K \alpha}$, respectively. The sharp rise in both profiles at the airsilicon critical angle ( $0.21 \mathrm{deg})$ indicates the high cleanliness of the exterior surface of the silicon layer. Figure $6 \mathrm{c}$ shows the associated depth profile for the four elements obtained with the same approach as for the Fe-implant above. The integrated areas under the fitted concentration profiles were $2.50 \pm 0.23 \times 10^{13} \mathrm{Ni} / \mathrm{cm}^{2}$ (Figure $5 \mathrm{c}$ green curve) and $2.46 \pm 0.22 \times 10^{13} \mathrm{Mn} / \mathrm{cm}^{2}$ (Figure 5c red curve) which are both consistent with the nominal values of $3 \times 10^{13}$ atoms $/ \mathrm{cm}^{2}$. The satisfactory results for the two implants containing Fe in sapphire and $\mathrm{Mn}$ and $\mathrm{Ni}$ in silicon-on-sapphire demonstrates the robustness of the analytical and modeling approach for determining fluences of implanted species.

\subsection{Silicon-on-Sapphire Solar Wind Flight Sample}

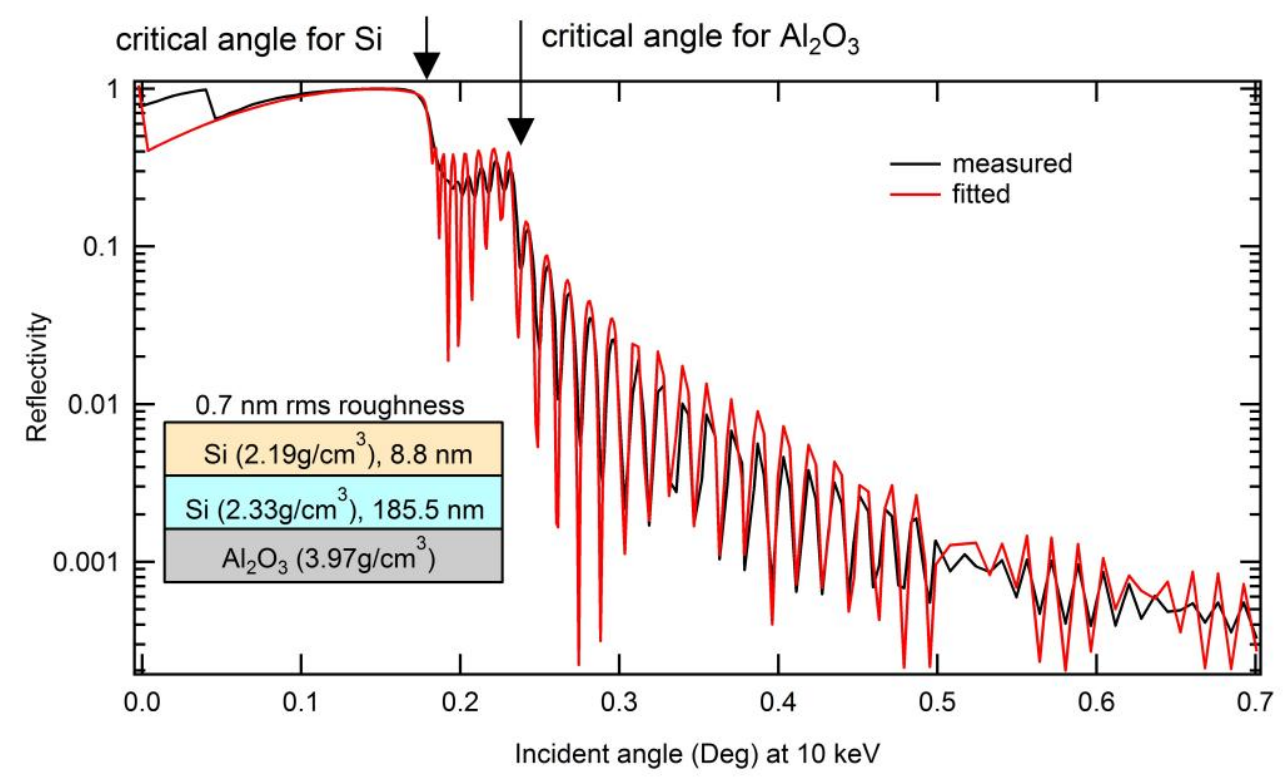

Figure 7: Measured reflectivity curve (black line) and fitted curve (red line) for Genesis flight SoS sample 60326. The optimized structural model is shown in the inset. 
The internal reference method was applied to determination of solar wind $\mathrm{Fe}$ and Ni fluences in Genesis silicon-on-sapphire flight sample SoS 60326. Figure 7 plots the
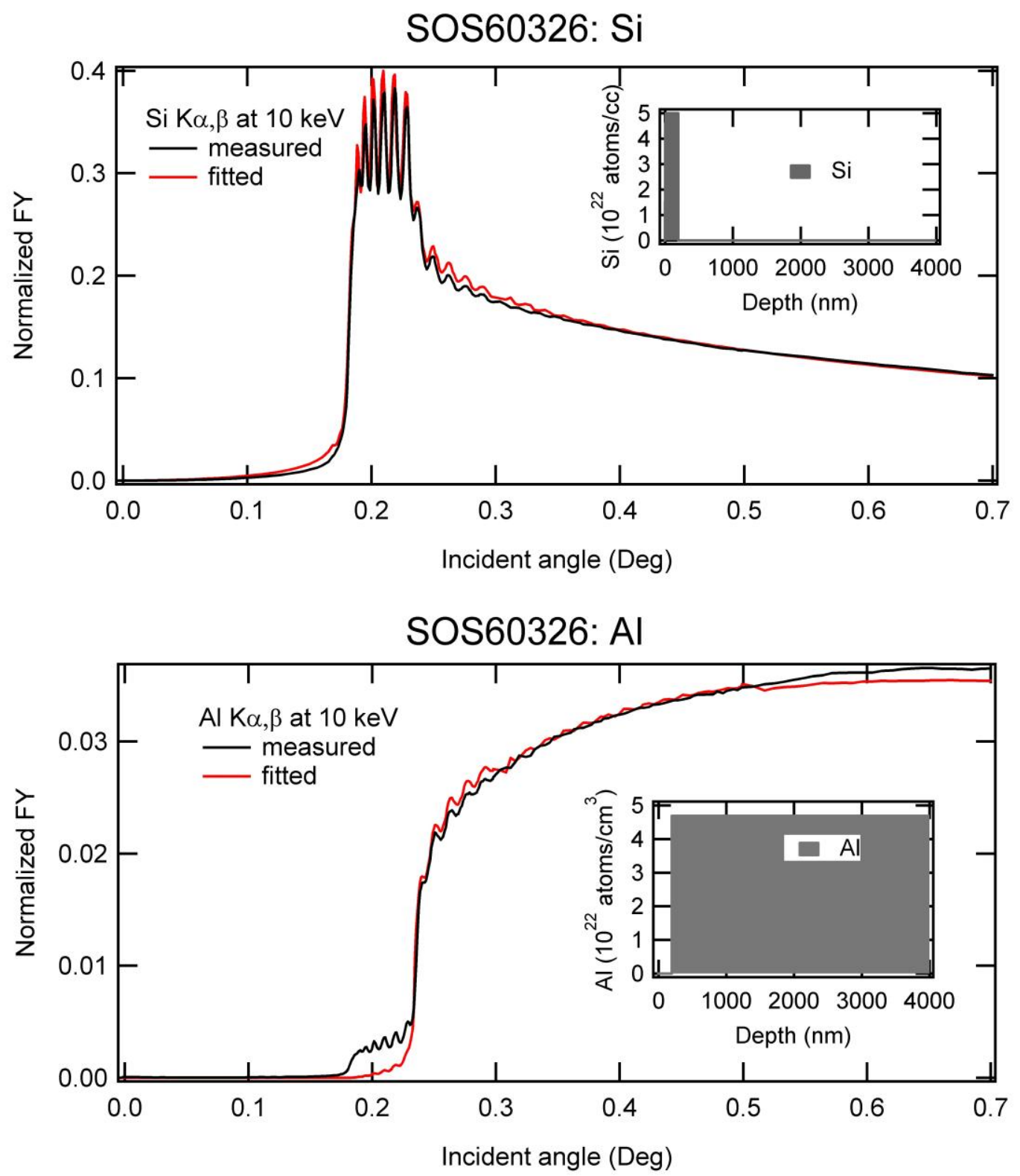

Figure 8: Measured (black) and best-fit (red) fluorescence yield curves for Si (top) and $\mathrm{Al}$ (bottom) in flight sample SoS60326. The fits assuming a structure of $200 \mathrm{~nm}$ silicon on top of a bulk sapphire substrate agree well with the measurements. These curves provide the internal referencing for obtaining quantitative elemental depth distributions for other elements. 
measured reflectivity curve (black line) and fitted curve (red line) showing good agreement between the two. The optimized model (inset) indicated $0.7 \mathrm{~nm}$ RMS surface roughness on the silicon, an $8.8 \mathrm{~nm}$ thick reduced-density silicon layer (density 2.19 $\mathrm{g} / \mathrm{cm}^{3}$ optimized), a bulk silicon coating layer $185.5 \mathrm{~nm}$ thick (density $2.33 \mathrm{~g} / \mathrm{cm}^{3}$ assumed) and the thick sapphire substrate (density $3.97 \mathrm{~g} / \mathrm{cm}^{3}$ assumed). The combined silicon layer thickness of $194 \mathrm{~nm}$ agrees well with the spectroscopic ellipsometry results on flight SoS wafers which indicated a Si layer of thickness $185 \mathrm{~nm}$ (McNamara and Stansbery 2005).

Figure 8 shows the measured (black) and best-fit (red) fluorescence yield curves for $\mathrm{Si}$ (top) and $\mathrm{Al}$ (bottom) in the flight sample SoS 60326. The fits assuming a structure of $200 \mathrm{~nm}$ silicon on top of a bulk sapphire substrate agree well with the measurements. These curves provide the internal referencing for obtaining quantitative elemental depth distributions for other elements, notably $\mathrm{Fe}$ and $\mathrm{Ni}$.

The top plot in Figure 9 shows the measured (black) and best-fit (red)

fluorescence yield curves for Fe. The bottom plot in Figure 9 shows the depth-dependent structure profile for Fe corresponding to the red fit in Figure 9 top. The fit was produced using two components, a Gaussian shaped surface component (Gaussian 1) and an asymmetric Gaussian shaped implant profile (Gaussian 2). The shape of the implant profile was constrained to be that measured by SIMS analysis for chromium as described above (equation 3 and Figure 2). Thus, the modeled depth profile for implanted Fe was constrained in width, asymmetry and depth of the maximum whereas the amplitude was the only variable. This approach assumes the depth distribution for $\mathrm{Cr}$ is the same as that 
for Fe which is a good assumption here since the integrated fluence results, the main goal of this work, are insensitive to the precise elemental depth distributions.
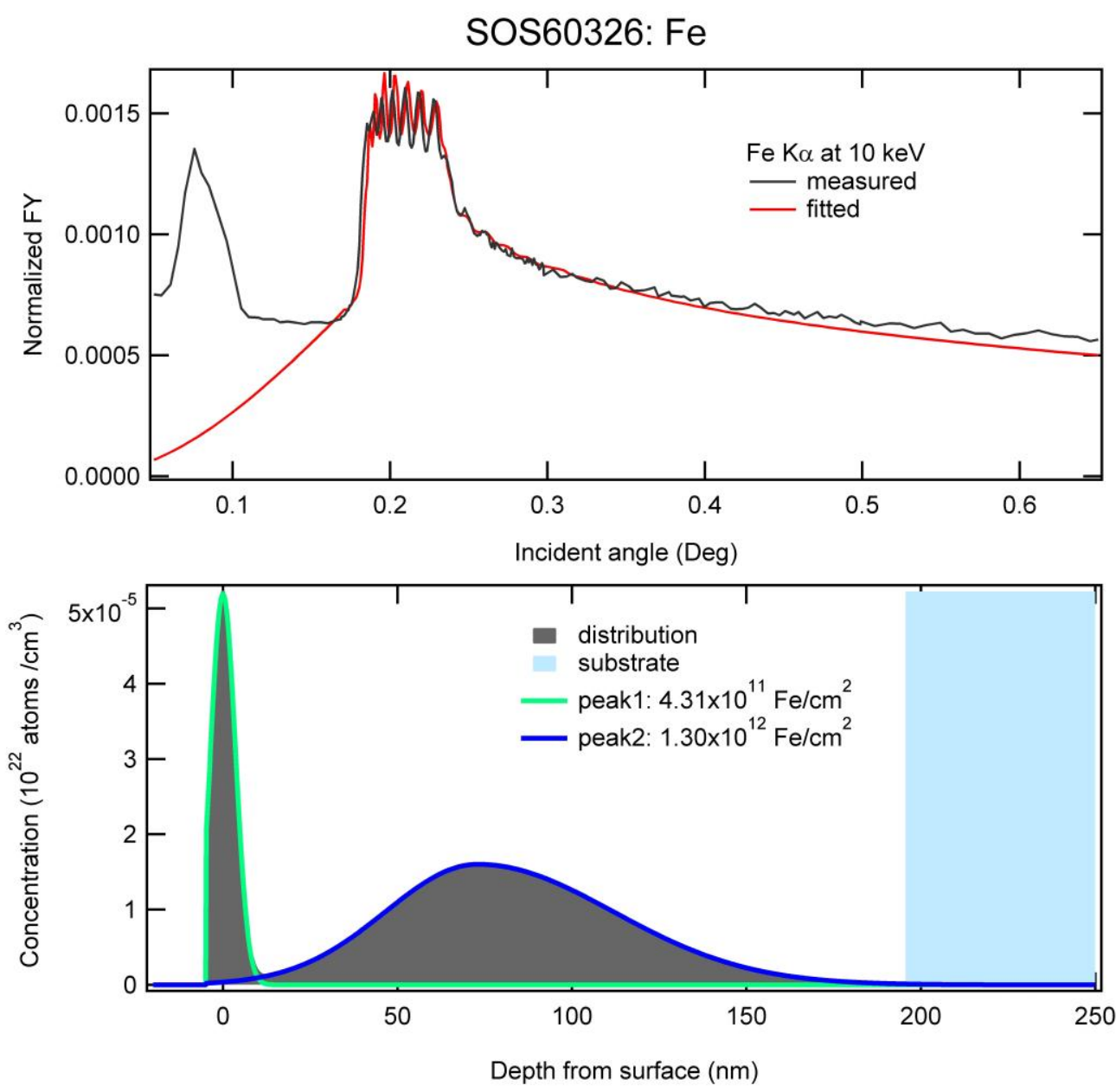

Figure 9: Top: Measured (black) and best-fit (red) fluorescence yield curves for Fe in flight sample SoS60326. The bottom plot shows the depth-dependent structure profile for Fe (gray area is the Fe distribution) corresponding to the red fit in the top plot. The fit was produced using two components, a Gaussian shaped surface component (Gaussian 1) and an asymmetric Gaussian shaped implant profile (Gaussian 2). The shape of the implant profile was constrained to be that measured by SIMS analysis for chromium (Fig. 2) as described in the text.

The results for Fe yield a surface-correlated (contamination) concentration of $4.31 \pm 0.40$ $\mathrm{x} 10^{11} \mathrm{Fe}$ atoms $/ \mathrm{cm}^{2}$ above a zone with concentration $1.30 \pm 0.12 \times 10^{12} \mathrm{Fe}$ atoms $/ \mathrm{cm}^{2}$, the latter being the solar wind implant. The uncertainty in the solar wind fluence was 
determined from the variance of the fit to the data with the majority of the uncertainty deriving from deconvolution of the significant surface contamination component.

Fe fluorescence detected below $\sim 0.18$ deg in the yield profile (black curve in Figure 9 top) suggests the presence of coarse, Fe-bearing, above-surface contamination which was under-fit by the 1- dimensional model (red curve in Figure 9 top). This lowangle misfit indicates that the Fe surface contamination determination (peak 1 in Figure 9 bottom inset) was underestimated but with negligible effect on the below-surface Fe implant determination.

Solar wind fluence for Ni in SoS 60326 was obtained in the same way as for Fe. The top plot in Figure 10 shows the measured (black) and best-fit (red) fluorescence yield curves for Ni. The bottom plot in Figure 10 shows the depth-dependent structure profile for Fe corresponding to the red fit in Figure 10 top. The results for Ni yielded a surfacecorrelated (contamination) concentration of $1.18 \pm 0.11 \times 10^{11} \mathrm{Ni}$ atoms $/ \mathrm{cm}^{2}$ and $2.34 \pm$ $0.22 \times 10^{11} \mathrm{Ni}$ atoms $/ \mathrm{cm}^{2}$, the latter being the solar wind implant. The modeled depth profile (Fig. 10 bottom) demonstrates that the measured $\mathrm{Ni}$ is distributed within the silicon layer and not concentrated at the $\mathrm{Si} /$ sapphire interface.

The Ni fluence determination was initially compromised by a detector artifact in the XRF spectra; the pile-up (or "sum”) peak from $\mathrm{Ca} \mathrm{K} \alpha$ (i.e., "Ca $\mathrm{K} \alpha+\mathrm{Ca} \mathrm{K} \alpha$ ”) produces an artifact peak at $7.38 \mathrm{keV}$ which is unresolvable from $\mathrm{Ni} \mathrm{K} \alpha(7.45 \mathrm{keV})$ considering the $\sim 0.15 \mathrm{keV}$ energy resolution of the energy dispersive detector. Consequently, SoS 60326 received an additional cleaning procedure to reduce the initial high level of $\mathrm{Ca}$ contamination on its surface. Interference from the $\mathrm{Ca} \mathrm{K} \alpha$ pile-up can be demonstrated to be negligible in the final dataset with the following argument. Since the 
pile-up probability is independent of energy, the " $\mathrm{Ca} \mathrm{K} \alpha+\mathrm{Ca} \mathrm{K} \alpha$ " intensity will be lower than the "Ca $\mathrm{K} \alpha+\mathrm{Si} \mathrm{K} \alpha$ " (5.4 keV) intensity by the intensity ratio of $\mathrm{Si} \mathrm{K} \alpha / \mathrm{Ca} \mathrm{K} \alpha$. In the $0.1 \mathrm{deg} \mathrm{XRF}$ spectrum, an angle at which the $\mathrm{Ca} \mathrm{K} \alpha$ intensity is maximum, $\mathrm{Ca} \mathrm{K} \alpha=1000$ cts (counts in central MCA channel), $\mathrm{Si} \mathrm{K} \alpha=8 \times 10^{5}$ cts and the $5.4 \mathrm{keV}$ peak
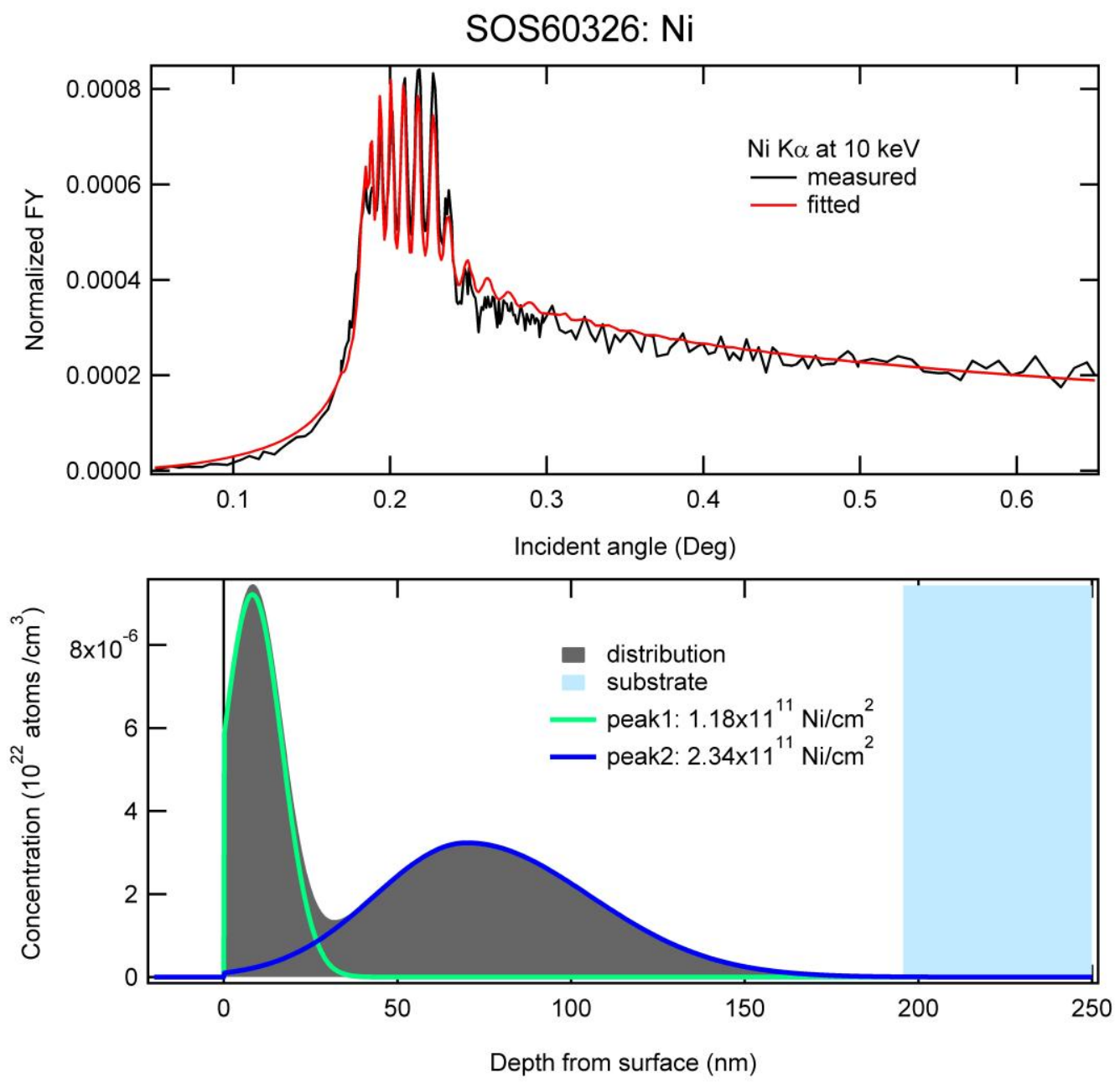

Fig. 10: Measured (black) and best-fit (red) fluorescence yield curves for $\mathrm{Ni}$ in flight sample SoS60326. The bottom plot shows the depth-dependent structure profile for Fe corresponding to the red fit in the top plot. The vertical line indicates the airsilicon interface. The fit was produced using two components, a Gaussian shaped surface component (Gaussian 1) and an asymmetric Gaussian shaped implant profile (Gaussian 2). The shape of the implant profile was constrained to be that measured by SIMS analysis for chromium (Fig. 2) as described in the text. 
$=100$ cts. Assuming the $5.4 \mathrm{keV}$ peak is all pile-up (a worse-case scenario since a $\mathrm{Cr} \mathrm{K} \alpha$ contribution is likely) produces an upper limit for the " $\mathrm{Ca} \mathrm{K} \alpha+\mathrm{Ca} \mathrm{K} \alpha$ " peak $=100 / 800=$ 0.8 cts. Since all Ni Ka peaks in the SoS 60326 spectra greatly exceed $1 \mathrm{ct}$, it is concluded that the pile-up interference is negligible.

The inferred solar wind fluences for $\mathrm{Fe}$ and $\mathrm{Ni}$ assume the epitaxial Si layer of the SoS collectors has negligible impurity concentrations of Fe and Ni. The flight criterion was for impurity levels to be $\leq 10 \%$ (and $\leq 1 \%$ if possible) of the anticipated 2-yr solar wind fluence for each element (Jurewicz et al. 2003; Burnett et al. 2003) although only a subset of elements (including $\mathrm{Fe}$ and $\mathrm{Ni}$ ) were verified to meet this criterion in at least one member of the collector set but not necessarily in silicon-on-sapphire collectors.

\section{Discussion}

Table 2 summarizes the fluence results from this work as well as other relevant results in the literature. Previously, we reported (Kitts et al. 2009) a Fe fluence result for a sapphire collector (50722) using a similar XSW experimental approach to that used here except that a synthesized Fe-implanted Genesis spare was used as an external standard, rather than using the internal reference method. That result, $1.6 \pm 0.4 \times 10^{12} \mathrm{Fe}$ atoms $/ \mathrm{cm}^{2}$, agrees well with the current result of $1.30 \pm 0.20 \times 10^{12} \mathrm{Fe}$ atoms $/ \mathrm{cm}^{2}$; the uncertainty is smaller in the latter because some sources of error are excluded when utilizing the internal standard method. The agreement between the Fe fluences for the two different collector materials (obtained in different experimental sessions) enhances the reliability of these results. If the two fluence values are treated as independent, the weighted mean is $1.36 \pm 0.18 \times 10^{12} \mathrm{Fe}$ atoms $/ \mathrm{cm}^{2}$. This value agrees well with the SIMS 
result of $1.41 \pm 0.08 \times 10^{12} \mathrm{Fe}$ atoms $/ \mathrm{cm}^{2}$ (Jurewicz et al. 2011) for silicon-on-sapphire, silicon, and diamond-like carbon collectors.

The pre-flight predicted Fe fluence was $1.7 \times 10^{12} \mathrm{Fe}$ atoms $/ \mathrm{cm}^{2}$ (Burnett et al. 2003) so the $x$-ray standing wave and SIMS results are in good agreement but about $20 \%$ lower than this value. As discussed by Jurewicz et al. (2011), the low Fe fluence might be explained by ion fractionation effects in the solar wind. Commonly discussed processes include Coulomb drag, first ionization potential (FIP) effects and first ionization time (FIT). Spacecraft studies show no evidence of significant fractionation for elements with FIP $<9 \mathrm{eV}$ such as Fe (Reisenfeld et al. 2007).

One way to test for fractionation effects is by comparing the concentrations of other elements. Jurewicz et al. (2011) specifically compared Fe with Mg from SIMS analyses, $\mathrm{Mg}$ is an element that has similar FIP near $8 \mathrm{eV}$ but much lower FIT than Fe, and found no obvious fractionation.

Nickel is an element that has similar FIP and FIT to those of Fe and measured fluences might be useful in identifying fractionation processes. The measured Ni fluence of $2.34 \pm 0.22 \times 10^{11} \mathrm{Ni}$ atoms $/ \mathrm{cm}^{2}$ is a factor of two higher than the expected fluence of $\sim 1 \times 10^{11} \mathrm{Ni}$ atoms $/ \mathrm{cm}^{2}$ (Burnett et al. 2003). However, FIP of Ni is essentially equal to that of FIP of Fe (7.9 vs 7.6, respectively) and Fe doesn't appear to be fractionated from photospheric. FIT requires a model with accompanying uncertainties, whereas FIP is an atomic constant.

Potential analytical reasons for over-estimation of the Ni solar wind fluence are discussed first. The possibility that the XRF sensitivity for Ni is too low can be ruled out by the facts that (1) Ni and Fe have very similar sensitivities and the Fe result is robust, 
and (2) the Ni concentration for the SoS implant is not overestimated. Another possibility is that the deconvolution of the surface-correlated (contamination) and implanted Ni has underestimated the amount of $\mathrm{Ni}$ in the former. This is highly unlikely based on the excellent fit obtained to the measured Ni fluorescence yield profile (Figure 10). A final possibility is that the $\mathrm{Ni}$ impurity concentration in the epitaxial silicon layer is comparable to the solar wind concentration. This might be ruled out because the selection criteria for the collector materials would preclude such high impurity levels, however, this possibility should be investigated further. There are a variety of potential contamination sources, including the post-flight cleaning process with ultra-pure water to remove Utah dust. However, the measured Fe fluences by multiple methods are reasonable so that any contamination mechanism would seem to require contamination by $\mathrm{Ni}$ and not Fe; not impossible but unlikely. The best way to evaluate the impurity level is through measurements on a SoS witness flag from the spacecraft. Unfortunately, the Utah impact prohibits the identification of such material. Next best is the analysis of SoS flight spares although the histories of these are not identical to those of the flight samples and a direct comparison may be difficult.

The measured $\mathrm{Ni} / \mathrm{Fe}$ is 0.17 which is a factor of $\sim 2.8$ higher than spacecraft solar wind measurements, photosphere observations, and compositions of meteorites (Karrer et al. 2007). This result is surprising in light of the expected absence of significant solar wind fractionation of these two elements based on similar CD, FIT and FIP parameters. Specifically, it suggests that more work should be done in establishing the $\mathrm{Ni}$ concentration in the as-manufactured epitaxial silicon layers of the SoS collectors. If the 
Ni fluence result proves to be robust, one interpretation is that there exists temporallyvariable $\mathrm{Fe}$ and $\mathrm{Ni}$ fractionation on the timescale of a year.

The internal reference method described here has the potential for providing solar wind fluence measurements of other elements, notably $\mathrm{Cr}$ and possibly Ca. Analyses of both of these elements are currently hindered by high Ca surface contamination. In measuring $\mathrm{Ca}$ itself, this contamination leads to uncertainties in deconvolution of implanted $\mathrm{Ca}$ from the high surface-correlated components. For $\mathrm{Cr}$, there is a potentially significant interference from the pile-up of "Si $\mathrm{K} \alpha+\mathrm{Ca} \mathrm{K} \alpha$ " at $5.43 \mathrm{keV}$ which nearly exactly overlaps the $\mathrm{Cr} \mathrm{K} \alpha$ peak at $5.41 \mathrm{keV}$. Improved cleaning procedures are likely to be needed to enable measurements on these elements.

\section{Conclusions}

X-ray standing wave fluorescence yield depth profiling was used to determine the solar wind implanted Fe and Ni fluences in a silicon-on-sapphire (SoS) Genesis collector (60326). An internal reference standardization method was developed based on fluorescence from $\mathrm{Si}$ and $\mathrm{Al}$ in the collector materials. Measured Fe fluence agreed well with that measured previously by us on a sapphire collector (50722) as well as SIMS results by Jurewicz et al. (2011). Measured Ni fluence was higher than expected by a factor of two; neither instrumental errors nor solar wind fractionation effects are considered significant perturbations to this value. Impurity $\mathrm{Ni}$ within the epitaxial $\mathrm{Si}$ layer remains a possibility that needs further investigation. If the measured Ni fluence is found to be robust, one interpretation is that there exists temporally-variable $\mathrm{Fe}$ and $\mathrm{Ni}$ fractionation on the timescale of a year. 


\section{Acknowledgements}

The contributions of Kathy Kitts (the PI on the NASA grants supporting this project) are particularly acknowledged. Dr. Kitts was involved in identifying appropriate samples for this work, collecting XSW data and reporting the initial results. The curatorial staff at Johnson Space Center is thanked for providing the flown samples and the Genesis team for providing the implant standards. The manuscript was improved by the valuable reviews of A. Jurewicz and an anonymous reviewer. This research was supported by NASA Grants DDAP No. NNX07AG02G and SRLIDAP No. NNX07AL96G to Northern Illinois University (K. Kitts, PI), and NASA LARS grants NNX10AH05G to Loyola University Chicago (M. Schmeling, PI) and NNH09AM48I (I. Veryovkin, PI) to Argonne National Laboratory. This work was performed at GeoSoilEnviroCARS (The University of Chicago, Sector 13), Advanced Photon Source (APS), Argonne National Laboratory. GeoSoilEnviroCARS is supported by the National Science Foundation - Earth Sciences (EAR-1128799) and Department of EnergyGeoSciences (DE-FG02-94ER14466). This research used resources of the Advanced Photon Source, a U.S. Department of Energy (DOE) Office of Science User Facility operated for the DOE Office of Science by Argonne National Laboratory under Contract No. DE-AC02-06CH11357.

\section{References}

Becker, R. S., J. A. Golovchenko, and J. R. Patel, "X-Ray evanescent-wave absorption and emission," Phys. Rev. Lett. 50,153-156 (1983). 
Bedzyk, M. J., D. H. Bilderback, G. M. Bommarito, M. Caffrey, and J. S. Schildkraut, "X-ray standing waves: a molecular yardstick for biological membranes," Science 241,1788-1791 (1988).

Bedzyk, M. J., G. M. Bommarito, and J. S. Schildkraut, "X-ray standing waves at a reflecting mirror surface," Phys. Rev. Lett. 62,1376-1379 (1989).

Bloch, J. M., M. Sansone, F. Rondelez, D. G. Peiffer, P. Pincus, M. W. Kim and P. M. Eisenberger, "Concentration profile of a dissolved polymer near the air-liquid interface: X-Ray fluorescence study," Phys. Rev. Lett. 54,1039-1042 (1985).

Burnett, D. S., "Solar composition from the Genesis Discovery Mission," Proceedings of the National Academy of Sciences 108, 19147-19151 (2011).

Burnett, D. S., "The Genesis solar wind sample return mission: Past, present, and future," Meteoritics \& Planetary Science 48, 2351-2370 (2013).

Burnett D. S., A. J. G. Jurewicz, D. S. Woolum, J. Wang, J. M. Paque, L. R. Nittler, K. M. McKeegan, M. Humayun, R. Hervig, V. S. Heber, and Y. Guan Y., "Ion implants as matrix-appropriate calibrators for geochemical ion probe analysis." Geostandards and Geoanalytical Research 39, 265-276 (2015).

Burnett, D. S., B. L. Barraclough, R. Bennett, M. Neugebauer, L. P. Oldham, C. N. Sasaki, D. Sevilla, N. Smith, E. Stansvery, D. Sweetnam, and R. C. Wiens, "The Genesis Discovery Mission: Return of solar matter to Earth,” Space Science Reviews 105, 509-534 (2003).

Calaway, M. J., D. S. Burnett, M. C. Rodriguez, S. Sestak, J. H. Allton, and E. K. Stansbery, E. K., "Decontamination uf Genesis array materials by UV ozone cleaning (abstract 1338)," $38^{\text {th }}$ Lunar and Planetary Science Conference (2007). 
Chantler, C. T., "Detailed tabulation of atomic form factors, photoelectric absorption and scattering cross section, and mass attenuation coefficients in the vicinity of absorption edges in the soft $\mathrm{x}$-ray $(\mathrm{Z}=30-36, \mathrm{Z}=60-89, \mathrm{E}=0.1 \mathrm{keV}-10 \mathrm{keV})$, addressing convergence issues of earlier work," J. Phys. Chem. Ref. Data 29, 597$1048,2000$.

Chantler, C. T., "Theoretical form factor, attenuation, and scattering tabulation for $\mathrm{Z}=1-$ 92 from E=1-10 eV to E=0.4-1.0 MeV," J. Phys. Chem. Ref. Data 24, 71-643, 1995.

De Boer, D. K. G., "Glancing-incidence x-ray fluorescence of layered materials," Phys. Rev. B 44, 498-511 (1991).

Dev, B. N., A. K. Das, S. Dev, D. W. Schubert, M. Stamm, and G. Materlik G., "Resonance enhancement of $\mathrm{x}$ rays in layered materials: Application to surface enrichment in polymer blends," Phys. Rev. B 61, 8462-8468 (2000).

Elam, W. T., B. D. Ravel, and J. R. Sieber, "A new atomic database for x-ray spectroscopic calculations," Radiat. Phys. Chem 63, 121-128, 2002.

Ghose, S. K., B. N. Dev, and A. Gupta, "Resonance enhancement of X-rays and fluorescence yield from marker layers in the thin films," Phys. Rev. B 64, 233403(1-4) (2001).

Gupta, A., P. Rajput, and C. Meneghini, "Depth-resolved x-ray absorption fine structure study of FeSi interfaces using x-ray standing waves," Phys. Rev. B 76,195401(18) (2007). 
Heber V.S.,K. D. McKeegan, D. S. Burnett, J. Duprat, Y. Guan, A. J. G. Jurewicz, C. T. Olinger, and S. P. Smith, "Accurate analysis of shallowly implanted solar wind ions by SIMS backside depth profiling.” Chemical Geology 390, 61-73 (2014).

Jurewicz, A. J. G., D. S. Burnett, D. S. Woolum, K. D. McKeegan, V. Heber, Y. Guan, M. Humayun, and R. Hervig, "Solar-wind Fe/Mg and a comparison with CI chondrites (abstract 1917)," 42 ${ }^{\text {nd }}$ Lunar and Planetary Science Conference (2011).

Jurewicz, A. J. G., D. S. Burnett, R. C. Wiens, T. A. Friedmann, C. C. Hays, R. J. Hohlfelder, K. Nishiizumi, J. A. Stone, D. S. Woolum, R. Becker, A. Butterworth, A. J. Campbell, M. Ebihara, I. A. Franchi, V. Heber, C. M. Hohenberg, M. Humayun, K. D. McKeegan, K. McNamara, A. Meshik, R. O. Pepin, D. Schlutter, and R. Wieler, "The Genesis solar-wind collector materials," Space Science Reviews 105, 535-560 (2003).

Karrer R., P. Bochsler, C. Giammanco, F. M. Ipavich, J. A. Paquette, and P. Wurz, "Nickel isotopic composition and nickel/iron ratio in the solar wind: Results from SOHO/CELIAS/MTOF,” Space Sci. Rev. 130, 317-321, 2007.

Kitts, K., Y. Choi, P. J. Eng, S. K. Ghose, S. R. Sutton, and B. Rout, “Application of grazing incidence x-ray fluorescence technique to discriminate and quantify implanted solar wind," J. Appl. Phys. 105, 064905(1-3) (2009).

Krause, M. O. and J. H. Oliver J. H., "Natural widths of atomic K and L levels, Ka X-ray lines and several KLL Auger lines,” J. Phys. Chem. 8, 329-338 (1979).

Krause, M. O., “Atomic radiative and radiationless yields for K and L shells," J. Phys. Chem. Ref. Data 8, 307-327, 1979. 
Lee, D. R., A. Hagman, X. Li, S. Narayanan, J. Wang, and K. R. Shull, "Perturbation to the resonance modes by gold nanoparticles in a thin-film-based x-ray waveguide," Appl. Phys. Lett. 88, 153101(1-3) (2006).

McMaster, W. H., N. Kerr Del Grande, J. H. Mallett, and J. H. Hubbell, "Compilation of X-ray cross sections," Lawrence Radiation Laboratory Report UCRL-5017 (National Bureau of Standards, Springfield, Virginia), 1969.

McNamara, K. M., and E. K. Stansbery E. K., “Analysis of molecular contamination on Genesis collectors through spectroscopic ellipsometry (abstract 2402)," $36^{\text {th }}$ Lunar and Planetary Science Conference (2005).

Parratt, L. G., "Surface studies of solids by total reflection of x-rays," Phys. Rev. 95, 359369 (1954).

Reisenfeld, D. B., D. S. Burnett, R. H. Becker, A. G. Grimberg, S. Heber, C. M. Hohenberg, A. J. G. Jurewicz, A. Meshik, R. O. Pepin, J. M. Raines, D. J. Schlutter, R. Wieler, R. C. Wiens, and T. H. Zurbuchen, "Elemental abundances of the bulk solar wind: Analyses from Genesis and ACE," Space Science Reviews 130, 79-86, 2007.

Sigmund, P., and A. Gras-Marti, "Distortion of depth profiles during sputtering: I. General description of collisional mixing," Nuclear Instruments and Methods 168, 389-394 (1980).

Templeton, A. S., T. P. Trainor, S. J. Traina, A. M. Spormann, and G. E. Brown, Jr., " $\mathrm{Pb}(\mathrm{II})$ distributions at biofilm-metal oxide interfaces," Proceedings of the National Academy of Sciences 98,11897-11902 (2001). 
Tiwari, M. K., G. M. Bhalerao, M. Babu, A. K. Sinha, and C. Mukherjee, "Investigation of metal nanoparticles on a Si surface using an X-ray standing wave field," $J$. Appl. Phys. 103, 054311(1-6) (2008).

Trainor, T. P., A. S. Templeton, and P. J. Eng, "Structure and reactivity of environmental interfaces: Application of grazing angle x-ray spectroscopy and long-period x-ray standing waves," J. Electron. Spectrosc. Relat. Phenom. 150, 66-85 (2006).

Veryovkin, I. V., C. E. Tripa, A. V. Zinovev, B. V. King, M. J. Pellin, and D. S. Burnett, "RIMS analysis of $\mathrm{Ca}$ and $\mathrm{Cr}$ in Genesis solar wind collectors," Surface and Interface Analysis 43(1-2), 467-469 (2011).

Wang, J., M. J. Bedzyk, and M. Caffrey, "Resonance-enhanced x-rays in thin films: A structure probe for membranes and surface layers," Science $\mathbf{2 5 8}$, 775-778 (1992). 
Table 1: Tabulated values for photo-electric cross-sections (in Barns/atoms) and fluorescence yield (unitless). $C_{I n t}$ is the product of the two. $C_{E x t}$ is the product of the transmission ratios from the sample surface through the fluorescence detector for each $\mathrm{K} \alpha$ radiation.

\begin{tabular}{|l|llll|}
\hline & $\mathrm{Al}$ & $\mathrm{Si}$ & $\mathrm{Cr}$ & $\mathrm{Fe}$ \\
\hline$\sigma_{\text {photo-e }}(7.6 \mathrm{keV})$ & 2615.0 & 3546.5 & 25213.7 & 31805.2 \\
$\omega_{\mathrm{K}}$ & 0.039 & 0.05 & 0.275 & 0.34 \\
\hline $\mathrm{C}_{\text {Int }}$ & 102.0 & 177.3 & 10813.8 & 6933.8 \\
$\mathrm{C}_{\text {Ext }}$ & 0.004822 & 0.03303 & 0.8802 & 0.9263 \\
\hline
\end{tabular}

Table 2: Summary of solar wind fluence results for $\mathrm{Fe}$ and $\mathrm{Ni}$

\begin{tabular}{|c|c|c|c|}
\hline & $\begin{array}{l}\text { Sample } \\
\text { number }\end{array}$ & $\begin{array}{l}\text { Fe fluences } \\
\left(\text { atoms } / \mathbf{c m}^{2}\right)\end{array}$ & $\begin{array}{l}\text { Ni fluences } \\
\left(\text { atoms } / \mathbf{c m}^{2}\right)\end{array}$ \\
\hline \multicolumn{4}{|l|}{ X-ray standing waves ${ }^{\#}$} \\
\hline Silicon-on-sapphire & 60326 & $1.30 \pm 0.10 \times 10^{12}$ & $2.34 \pm 0.22 \times 10^{11}$ \\
\hline Sapphire* & 50722 & $1.6 \pm 0.4 \times 10^{12}$ & NA \\
\hline Two-collector mean & & $1.36 \pm 0.18 \times 10^{12}$ & NA \\
\hline $\mathrm{Ni} / \mathrm{Fe}$ & & 0.17 & \\
\hline SIMS $^{\wedge}$ & & $1.41 \pm 0.08 \times 10^{12}$ & NA \\
\hline Predicted fluences $^{\$}$ & & $1.7 \times 10^{12}$ & $1.0 \times 10^{11}$ \\
\hline
\end{tabular}

\# This work

* Kitts et al. 2009

$\wedge$ Jurewicz et al. 2011

\$ Burnett et al. 2003

$\mathrm{NA}=$ not available 